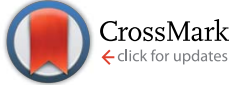

Cite this: RSC Adv., 2016, 6, 70394
Received 15th June 2016

Accepted 19th July 2016

DOI: 10.1039/c6ra15610j

www.rsc.org/advances

\section{Surface and interface interplay on the oxidizing temperature of iron oxide and Au-iron oxide core-shell nanoparticles $\uparrow$}

\author{
Sarveena, ${ }^{\text {a Diego Muraca, }}{ }^{\text {bh }}$ P. Mendoza Zélis, ${ }^{c}$ Y. Javed, ${ }^{d}$ N. Ahmad, ${ }^{\text {e }}$ J. M. Vargas, ${ }^{f}$ \\ O. Moscoso-Londoño, ${ }^{b}$ M. Knobel, ${ }^{\text {bi }}$ M. Singh ${ }^{a}$ and S. K. Sharma*ga
}

\begin{abstract}
This article presents the effect of oxidation temperature on shape anisotropy, phase purity and growth of core-shell heterostructures and consequently their effect on structure-property relationships. Iron oxide and Au-iron oxide nanocomposites were synthesized by a thermal decomposition method by passing pure oxygen at different temperatures $\left(125-250^{\circ} \mathrm{C}\right)$. The prepared nanoparticles were surface functionalized by organic molecules; the presence of the organic canopy prevented both direct particle contact as well as further oxidation, resulting in the stability of the nanoparticles. We have observed a systematic improvement in the core and shell shape through tuning the reaction time as well as the oxidizing temperatures. Spherical and spherical triangular shaped core-shell structures have been obtained at an optimum oxidation temperature of $125^{\circ} \mathrm{C}$ and $150{ }^{\circ} \mathrm{C}$ for 30 minutes. However, further increase in the temperature as well as oxidation time results in core-shell structure amendment and results in fully grown core-shell heterostructures. As stability and ageing issues limit the use of nanoparticles in applications, to ensure the stability of the prepared iron oxide nanoparticles we performed XRD analysis after more than a year and they remained intact showing no ageing effect. Specific absorption rate values useful for magnetic fluid hyperthermia were obtained for two samples on the basis of detailed characterization using $\mathrm{X}$-ray diffraction, high-resolution transmission electron microscopy, Mössbauer spectroscopy, and dc-magnetization experiments.
\end{abstract}

\section{Introduction}

Nanocomposites that combine the distinct properties of the diverse constituents in a single structure have a plethora of applications in the myriad fields of technology due to their combined physicochemical properties. They are widely used in

${ }^{a}$ Department of Physics, H. P. University, Shimla, 171005, HP, India

${ }^{b}$ Instituto de Física Gleb Wataghin, Universidade Estadual de Campinas (UNICAMP), 13083-859 SP, Brazil

'Instituto de Física de La Plata (IFLP-CONICET), Departamento de Física, Facultad de Ciencias Exactas, Universidad Nacional de La Plata (UNLP), c.c. 67, 1900 La Plata, Argentina

${ }^{d}$ Department of Physics, University of Agriculture, Faisalabad, Pakistan

${ }^{e}$ Laboratoire Matériaux et Phénomènes Quantiques, UMR 7162 CNRS/Université Paris Diderot, 10 rue Alice Domon et Léonie Duquet, Paris F-75205 Cedex 13, France

${ }^{f}$ Centro Atomico Bariloche (CNEA), Instituto Balseiro (U. N. Cuyo) and Conicet, 8400 San Carlos de Bariloche, Río Negro, Argentina

${ }^{g}$ Departamento de Física, Universidade Federal do Maranhão, 65085-580 São Luis, Brazil. E-mail: surender76@gmail.com

${ }^{h}$ Centro de Ciencias Naturais e Humanas, Universidade Federal do ABC-UFABC, Av. dos Estados, 5001, Santo André, Brazil

${ }^{i}$ Laboratório Nacional de Nanotecnologia (LNNAno/CNPEM), Rua Giuseppe Máximo Scolfaro 10000, 13083-100, Campinas-SP, Brazil

$\dagger$ Electronic supplementary information (ESI) available. See DOI: 10.1039/c6ra15610j the areas of electronics, photonics, catalysis, biotechnology, and nanotechnology. Core-shell and exchange-coupled magnetic nanosystems are two well-known composite systems that have shown enhanced optical, magnetic, and catalytic properties compared to their individual single-component materials. ${ }^{1}$ From this viewpoint, magnetic composite structures based upon nanometer-sized iron oxide (IO) and gold particles (Au) have a lot of potential as due to their intrinsic magnetic features combined with the nanosized and surface effects; and gold nanoparticles are widely utilized in many standard tools for measuring adsorption, due to their intense surface plasmon resonance., ${ }^{2,3}$ Iron oxide nanoparticles are promising new tools for controlled release of drugs because they can satisfy the two most important criteria for successful therapy, i.e., spatial placement and temporal delivery. ${ }^{4}$ The coupling of plasmonic effect of gold nanoparticles with magnetic properties of iron oxide nanoparticles has shown great promise as bi-functional agents in biomedical applications, such as integrated imaging, diagnosis, targeted delivery, and photothermal therapy. ${ }^{5}$ The deposition of metal oxides onto noble metals significantly affects their optical properties-as the plasmon resonance frequency is shifted. This may be utilized to tune the frequency of plasmon resonances, and to control plasmon-exciton interactions between semiconductors and noble metals in multicomponent periodic 
arrays. ${ }^{6}$ The layer of iron oxide on the gold makes these new coreshell NPs amenable for use in magnetic separation, yet it does not block the electromagnetic effect for the enhancement of the vibrational modes of the molecule adsorbed on the NP surface. ${ }^{7}$ Enhanced magneto-optical signals have been reported for several noble-metal/ferrites and attributed to optical amplification produced by the giant electromagnetic field associated with the SPR (surface plasmon resonance) in the plasmonic neighbor. ${ }^{8}$ This new material would contribute to the growing class of multifunctional NPs. Therefore, the thriving combination of gold and iron oxide multifunctional nanohybrids promise to combine the unique traits of both gold and iron oxide to enable new applications. Core-shell nanoparticles with magnetic core and non-magnetic shell components e.g. $\mathrm{Fe}_{3} \mathrm{O}_{4} @ \mathrm{Au}$ are well studied system in recent years, ${ }^{\mathbf{9}, 10}$ however, magnetic properties of the inverse structure, namely, nanoparticles with a non-magnetic core and a magnetic shell, have rarely been studied. So, in this paper we have made an attempt to study structure-property relationship of iron oxide and inverse Au-iron oxide core/shell system synthesized using chemical route.

A number of methods have been developed for synthesizing magnetic nanoparticles with different compositions. ${ }^{\mathbf{1 1}, \mathbf{1 2}}$ The coating of metallic nanoparticles with a thin shell of a magnetic material is synthetically challenging, and only a few reports demonstrate this possibility. ${ }^{\mathbf{1 3}}$ Here we report a facile and reproducible chemical synthesis of iron oxide (IO) and $\mathrm{Au}$-iron oxide (AIO) nanocomposites by thermal decomposition method with controllable morphology of the outer oxide shell. To investigate structure-property relationships as a function of the synthesis procedure, as well as to compare the structural and magnetic properties among the hybrid nanosystems (Au@iron oxide) and their solely magnetic component (iron oxide), the prepared nanocomposites were characterized by X-ray diffraction (XRD), small angle X-ray scattering (SAXS), high-resolution transmission electron microscopy (HRTEM), dc-magnetization using a Magnetic Property Measurement System from Quantum Design (MPMS), room temperature ${ }^{57} \mathrm{Fe}$ Mössbauer spectroscopy and time dependent calorimeteric measurements. We have analyzed the different aspects of our synthesis approach with varying oxidation temperature and discuss the magnetic properties of these nanocomposites. The surface of iron oxide nanoparticles was functionalized by organic molecules to prevent both direct particle contact as well as their further oxidation. Aging is one of the most important and potentially limiting factors in the use of iron oxide nanoparticles in various applications. We have also monitored the aging of iron oxide nanoparticles for a year to ensure the stability of our system and the results were surprising showing no ageing effect. The applicability of prepared nanocomposites for hyperthermia application was investigated by calculating SAR values for two samples with different shape and morphology.

\section{Experimental section}

\subsection{Materials}

Iron acetylacetonate $\left(\mathrm{Fe}(\mathrm{acac})_{3}, 99.9 \%\right)$, gold(III) chloride trihydrate $\left(\mathrm{HAuCl}_{4} \cdot 3 \mathrm{H}_{2} \mathrm{O}, \geq 49.0 \%\right.$ Au basis), oleic acid (techn. 90\%), 1,2-hexadecanediol (techn. 90\%), 1-octadecene (techn. 90\%) were purchased from Aldrich and used without further purification. Oleylamine (80-90\%) was obtained from ACROS.

\subsection{Synthesis of iron oxide (IO) nanoparticles}

The iron oxide and Au-iron oxide core-shell nanoparticles were prepared by chemical route published elsewhere. ${ }^{14}$ IO nanoparticles with sizes in the range 13-42 $\mathrm{nm}$ were synthesized using thermal decomposition of $\mathrm{Fe}(\mathrm{acac})_{3}(2.0 \mathrm{mmol})$ in the presence of 1-octadecene $(20 \mathrm{ml})$ and the surfactants oleic acid $(8 \mathrm{ml})$ and oleylamine $(12 \mathrm{ml})$. The above mixture was gently heated at $120{ }^{\circ} \mathrm{C}$ for 60 minutes under continuous Ar flux with continuous stirring. The above mixture was slowly heated up to a final temperature of $315^{\circ} \mathrm{C}$ for 60 minutes under Ar flux with heating rate of $\sim 6^{\circ} \mathrm{C} \mathrm{min}^{-1}$. The solution was then cooled down to different temperature, $T_{\text {cooling }}$ where $T_{\text {cooling }}=125^{\circ} \mathrm{C}, 150{ }^{\circ} \mathrm{C}$, $200{ }^{\circ} \mathrm{C}$, and $250{ }^{\circ} \mathrm{C}$ for 30 minutes with the passage of pure oxygen. Finally, the solution was cooled to room temperature and the nanoparticles were washed adding access of ethanol and centrifugation at $25000 \mathrm{rpm}$ for 20 minutes. This procedure was repeated 3-4 times by dispersing the nanoparticles in toluene, adding excess of ethanol and centrifugation. Finally, the nanoparticles were dispersed in toluene (concentration of $0.05 \mathrm{~g} \mathrm{ml}^{-1}$ ) with 2-3 drops of oleylamine for the long-term storage.

\subsection{Synthesis of Au nano seeds}

To synthesize $\mathrm{Au}$ nanoparticles, we have adopted a procedure reported elsewhere with slight modifications. ${ }^{15} 50 \mathrm{mg}$ of $\mathrm{HAuCl}_{4} \cdot 3 \mathrm{H}_{2} \mathrm{O}$ was dissolved in $20 \mathrm{ml}$ oleylamine by using sonication at room temperature. The clear, orange-yellow solution was then transferred to $100 \mathrm{ml}$ of three-neck flask equipped with thermometer and reflux condenser. Under $\mathrm{Ar}$ flux, the solution temperature was increased to $125^{\circ} \mathrm{C}$; gradually forming a pale yellow solution that finally became deep purple in color. The solution was kept at this temperature for 25-30 minutes and then allowed to cool to the room temperature. The formed $\mathrm{Au}$ nanoparticles were collected by adding access of ethanol and followed by centrifugation as described above. This procedure was repeated 3-4 times by dispersing the nanoparticles in toluene, adding excess of ethanol and centrifugation. Finally, the nanoparticles were dispersed in toluene (concentration of $0.05 \mathrm{~g} \mathrm{ml}^{-1}$ ) with 2-3 drops of oleylamine for the long-term storage.

\subsection{Synthesis of Au-iron oxide (AIO) nanoparticles}

Using our previous method described above for the synthesis of IO nanoparticles, $\mathrm{Fe}(\mathrm{acac})_{3}(2.0 \mathrm{mmol})$ was mixed with 1-octadecene $(20 \mathrm{ml})$ in the presence of surfactants oleic acid $(8 \mathrm{ml})$ and oleylamine $(12 \mathrm{ml})$ and heated slowly from room temperature to $120^{\circ} \mathrm{C}$ for 60 minutes. At this temperature, $33 \mathrm{mg}$ of the as synthesized $\mathrm{Au}$ nano seeds were mixed with $3 \mathrm{ml}$ of toluene and added slowly and drop wise on the previous solution to stay there for another 5 minutes. The solution temperature then increased to $315{ }^{\circ} \mathrm{C}$ at a rate of $6{ }^{\circ} \mathrm{C} \mathrm{min}^{-1}$ and stayed there for 60 minutes under Ar flux. The solution was then cooled down to 
different cooling temperature, $T_{\text {cooling }}$ where $T_{\text {cooling }}=125{ }^{\circ} \mathrm{C}$, $150{ }^{\circ} \mathrm{C}, 200{ }^{\circ} \mathrm{C}$, and $250{ }^{\circ} \mathrm{C}$ for 30 minutes with the passage of pure oxygen. Finally, the solution was cooled to room temperature and washed and stored following the same procedure described above.

\subsection{Iron oxide nanoparticle characterizations}

The prepared nanocomposites were characterized for its structural and magnetic properties. The particle diameter and its distribution were acquired by means of transmission electron microscopy (TEM) (200 keV JEM 2010 microscope) by drying a toluene dispersion of the nanoparticles on a carbon coated copper grid and allowing it to dry. The structure was determined by X-ray diffraction (XRD) (Philips, X-PERT) with Co $\mathrm{K}_{\alpha}$ radiations. For small angle X-ray scattering (SAXS) measurements, the IO samples were prepared in a colloidal solution of toluene at $1: 20$ volume concentration, the obtained SAXS data were collected at the Brazilian Synchrotron laboratory and analyzed assuming a polydisperse spherical form factor and a structure factor in order to take into account the aggregate effects. The magnetic properties were measured on dried powder sample using MPMS magnetometer with fields up to $20 \mathrm{kOe}$ and temperatures from $2-350 \mathrm{~K}$. The zero field cooling (ZFC) and field cooling (FC) measurements were carried out as follow: the sample was first cooled down from $350 \mathrm{~K}$ to $5 \mathrm{~K}$ in a zero magnetic field, then a static magnetic field of $H=50$ Oe was applied, and $M_{\mathrm{ZFC}}$ was measured during warming up from $5 \mathrm{~K}$ to $350 \mathrm{~K}$; finally the sample was cooled down to $5 \mathrm{~K}$ under the same field $H$, and $M_{\mathrm{FC}}$ was measured during the cooling cycle. The $14.4 \mathrm{keV}\left(I_{\mathrm{g}}=1 / 2, M_{1}, I_{\mathrm{e}}=3 / 2\right){ }^{57} \mathrm{Fe}$ Mössbauer spectra were acquired using a $20 \mathrm{mCi}{ }^{57} \mathrm{Co}(\mathrm{Rh})$ source at $300 \mathrm{~K}$ in transmission geometry, with a standard spectrometer operating in the constant acceleration regime. The isomer shifts were measured in relation to $\alpha$-Fe at $300 \mathrm{~K}$. The number of channels used for transmission data recording versus source-absorber velocity was 1024 . The spectrometer line width with a $13 \mu \mathrm{m}$ $\alpha$-Fe absorber was $0.21 \mathrm{~mm} \mathrm{~s}^{-1}$. Specific absorption rate (SAR) values were determined through time-dependent calorimetric measurements. SAR experiments were conducted in a clear Dewar glass containing $0.5 \mathrm{ml}$ of ferrofluid (FF) located at the center of a 5 turns duty coil ( $25 \mathrm{~mm}$ inner diameter). The coil was fed with a $255 \mathrm{kHz}$ ac current generating an alternating magnetic field with amplitudes $\left(H_{0}\right)$ of $440 \mathrm{Oe}$. The temperature was determined with optic fiber sensors in contact with the FF and connected to a calibrated signal conditioner (Neoptix) with an accuracy of $0.1{ }^{\circ} \mathrm{C}$. The FF temperature was kept below $30{ }^{\circ} \mathrm{C}$ in order to minimize the fluid's evaporation and prevent its destabilization.

\section{Results and discussion}

\subsection{Iron oxide nanoparticles: structural and magnetic characterizations}

Fig. 1a-d shows the representative TEM images for the IO nanoparticles oxidized at $125{ }^{\circ} \mathrm{C}, 150{ }^{\circ} \mathrm{C}, 200{ }^{\circ} \mathrm{C}$ and $250{ }^{\circ} \mathrm{C}$ for 30 minutes during cooling from above the final refluxing

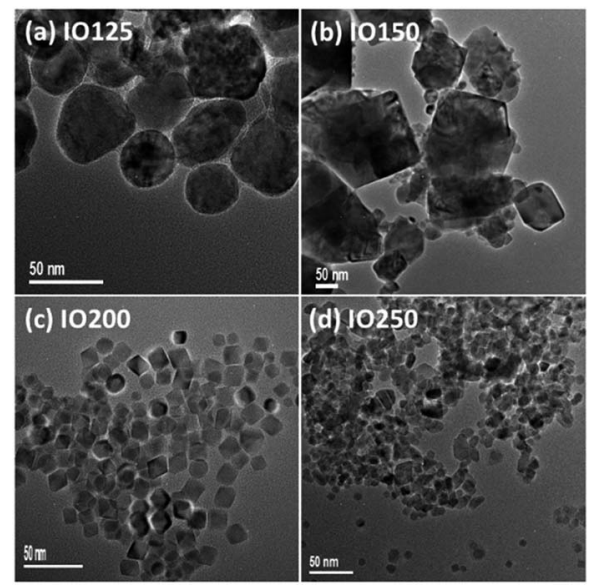

Fig. 1 TEM images of iron oxide nanoparticles (IONPs) oxidized at (a) $125^{\circ} \mathrm{C}$, (b) $150^{\circ} \mathrm{C}$, (c) $200^{\circ} \mathrm{C}$, and (d) $250^{\circ} \mathrm{C}$ for 30 minutes during cooling from $315^{\circ} \mathrm{C}$.

temperature at $315{ }^{\circ} \mathrm{C}$. Clearly one can see a noticeable difference in both sizes as well as in the shapes of the synthesized nanoparticles. The lattice and crystallinity of the IO nanoparticles has been further studied based on the HRTEM images collected over the various parts (see Fig. 2). The corresponding high resolution-TEM image for the IO125 shows that it is highly crystallized, elongated spherical shape nanoparticles as indicated by apparent atomic lattice fringes (Fig. 2a) as well as the presence of lower contrast shell surrounding the darker core (yellow dotted lines). Atomic fringes of $d_{110}=0.201 \mathrm{~nm}$, and $d_{311}=0.272 \mathrm{~nm}$ were observed in the inner part (core) and outer (shell), respectively, which corresponds to $\mathrm{Fe}(110)$, and $\mathrm{Fe}_{x} \mathrm{O}_{y}(311)$ atomic spacing, respectively.

For IO150, the size and shape changed completely from elongated spherical to small/irregular sheets, but with nonuniformity in contrast variations. A careful analysis of the

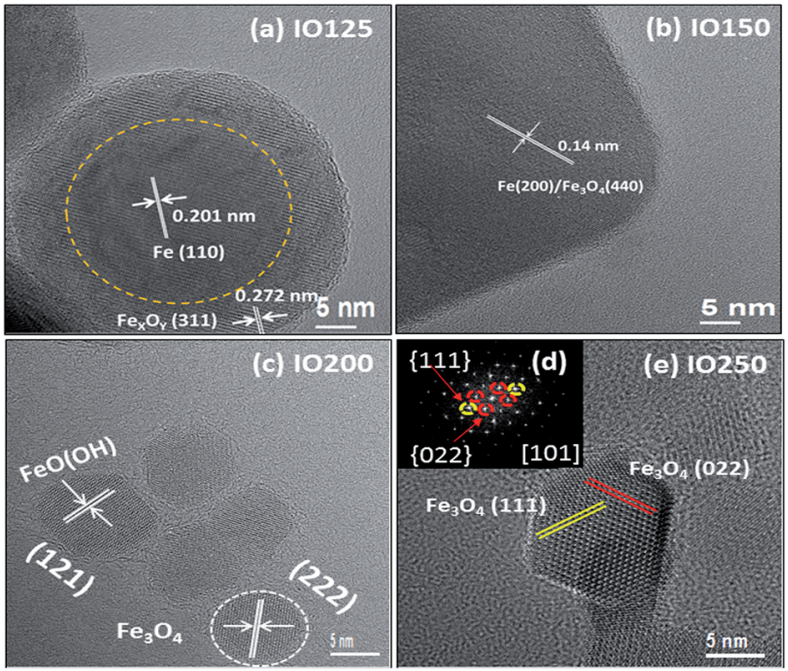

Fig. 2 HRTEM images of iron oxide nanoparticles (IONPs) oxidized at (a) $125^{\circ} \mathrm{C}$, (b) $150{ }^{\circ} \mathrm{C}$, (c) $200^{\circ} \mathrm{C}$, and (d and e) $250{ }^{\circ} \mathrm{C}$ for 30 minutes during cooling from $315^{\circ} \mathrm{C}$. 
atomic fringes corresponds to $\mathrm{Fe}(200)$, and $\mathrm{Fe}_{3} \mathrm{O}_{4}(440)$ without any signature of wüstite phase. Interestingly for IO200, the morphology of the nanoparticles is entirely different including good crystallinity spherical, triangular, capsule, rectangle and hexagonal shapes. The corresponding HRTEM shows well developed lattice fringes extending throughout the particle in the form of capsule, rectangle, triangle, spherical shapes and so on hence confirming the monocrystalline nature of the individual particles (see Fig. 2c). The distance between adjacent lattice fringes measured were $\sim 0.21 \mathrm{~nm}$ and $\sim 0.16 \mathrm{~nm}$ correspond to the (140) and (151) reflections of $\alpha-\mathrm{FeO}(\mathrm{OH}), \sim 0.23 \mathrm{~nm}$ and $\sim 0.13 \mathrm{~nm}$ corresponds to the (121) and (533) reflections of $\alpha-\mathrm{FeO}(\mathrm{OH})$ and $\mathrm{Fe}_{3} \mathrm{O}_{4}$, respectively (see ESI $\dagger$ ).

We have also observed an atomic lattice spacing of $\sim 0.18 \mathrm{~nm}$ for triangular shaped particle, $\sim 0.25 \mathrm{~nm}$ for spherical one, which belongs to $\alpha-\mathrm{FeO}(\mathrm{OH})$ and $\mathrm{Fe}_{3} \mathrm{O}_{4}$ respectively. Indeed, it has been found that the spherical shaped particles belongs to either iron oxide (magnetite or maghemite), whereas the capsule, rectangle, triangle and any other irregular shaped particle belongs to $\alpha-\mathrm{FeO}(\mathrm{OH})$ phase.

Finally, for IO250, again we found highly crystalline iron oxide nanoparticles with various shape as obtained as for IO200, but without any traces of $\alpha-\mathrm{FeO}(\mathrm{OH})$. Thus, the oxidizing temperature during cooling is clearly affecting the shape, size as well as the phase composition of the iron oxide nanoparticles. At low oxidizing temperatures, there is a mixture of $\alpha$-Fe, $\alpha$ $\mathrm{FeO}(\mathrm{OH})$, and $\mathrm{Fe}_{x} \mathrm{O}_{y}$, but at the high temperature, the most probable phase of iron oxide is either $\mathrm{Fe}_{3} \mathrm{O}_{4}$ or $\gamma-\mathrm{Fe}_{2} \mathrm{O}_{3}$. The detailed explanation of this issue is however beyond the scope of this paper, where more systematic experiments at different stages are required. However, the approach is promoting a single-step process of nucleation and growth of nanoparticles, which means control over the size, shape as well as phase purity of iron oxide nanoparticles.

The phase composition and crystal structure has been further investigated by X-ray diffraction technique. Fig. 3 shows the powder XRD patterns for the IO nanoparticles oxidized at different temperatures for 30 minutes during cooling from 315 ${ }^{\circ} \mathrm{C}$. Influence of oxidation temperatures can be clearly seen on the phase composition and structure of iron oxide nanoparticles. For all the samples, the XRD analysis shows the formation of mixed iron oxide phases. The pattern for IO125 consists of diffraction peaks arising from the three major phases: $\alpha$-Fe (JCPDF \# 85-1410), FeO (JCPDF \# 77-2355) and spinel iron oxide $\left(\mathrm{Fe}_{3} \mathrm{O}_{4} \mathrm{JCPDF}\right.$ \# 85-1436 and/or $\gamma$ - $\mathrm{Fe}_{2} \mathrm{O}_{3} \mathrm{JCPDF} \#$ 391346). The estimated amount of $\alpha$-Fe for IO125 is $\sim 6.9(2) \%$. However, increasing the oxidizing temperature to $150{ }^{\circ} \mathrm{C}$, we observed that the wüstite $(\mathrm{FeO})$ phase is completely washed out, and at the same time the amount of $\alpha$-Fe phase increased from $6.9(2)$ to $11.8(2) \%$. Indeed wüstite and spinel type iron oxide phases such as $\mathrm{Fe}_{3} \mathrm{O}_{4}$ and $\gamma-\mathrm{Fe}_{2} \mathrm{O}_{3}$ have face centered cubic (fcc) oxygen sub-lattice and hence yields similar X-ray diffraction patterns, however their major peaks have somewhat at different positions. Here we would like to mention that the dominating phase for samples IO125 and IO150 is spinel iron oxide $\left(\mathrm{Fe}_{3} \mathrm{O}_{4} /\right.$ $\gamma-\mathrm{Fe}_{2} \mathrm{O}_{3}$ ) with minor peaks of $\alpha$-Fe and $\mathrm{FeO}$, wüstite (only in case of IO125) around the major one. On the other hand for IO200,

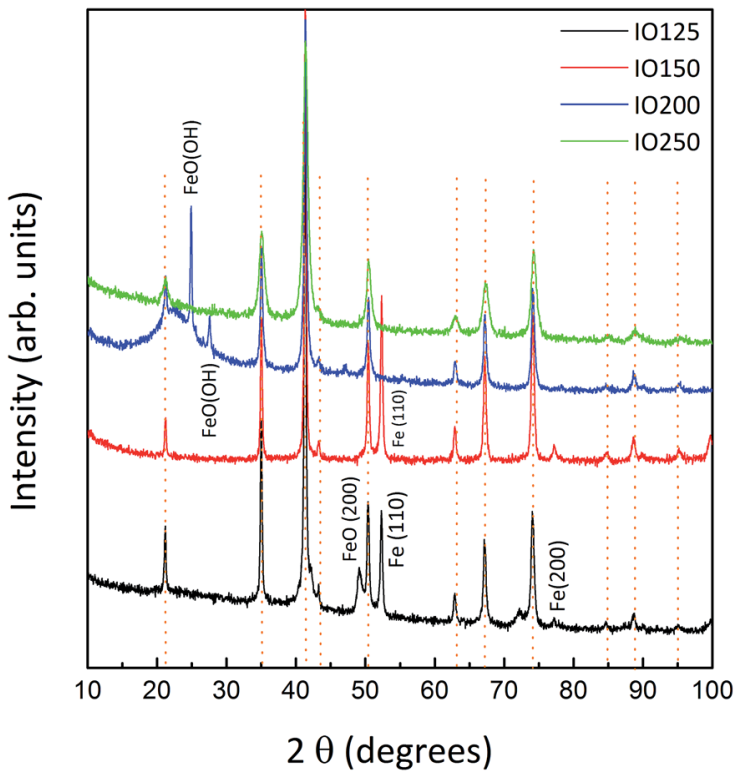

Fig. 3 X-ray diffraction (XRD) patterns of the iron-oxide nanoparticles oxidized at $125^{\circ} \mathrm{C}, 150{ }^{\circ} \mathrm{C}, 200^{\circ} \mathrm{C}$ and $250^{\circ} \mathrm{C}$ for 30 minutes during cooling from $315^{\circ} \mathrm{C}$.

we observed a mixed phase of dominating spinel iron oxide with traces of $\alpha-\mathrm{FeO}(\mathrm{OH})$ goethite (JCPDF \# 01-0463). Here we would like to mention that increasing the oxidizing temperature from $200{ }^{\circ} \mathrm{C}$ to $250{ }^{\circ} \mathrm{C}$; we observed a spinel iron oxide phase consisting of $\mathrm{Fe}_{3} \mathrm{O}_{4} / \gamma-\mathrm{Fe}_{2} \mathrm{O}_{3}$. In brief, the oxidizing the iron oxide nanoparticles during cooling from above the refluxing temperature is a decisive parameter and playing an important role on the phase composition as well as shape/size of the iron oxide nanoparticles.

Moreover, the effective crystallite size was estimated using the Scherrer formula ${ }^{16}$ by taking the full width at half maximum (FWHM) of the most intense peak obtained from the pattern (see ESI Table $3 \dagger$ ). The resulting crystallite size values and the observed lattice parameters are tabulated in the ESI. $\dagger$ It is observed that the reflections coming from the samples oxidized at higher temperature were broadened suggesting the small size of crystallite as is confirmed by crystallite size calculated by Scherrer formula. A careful investigation of these reflections shows that they actually become broaden non-uniformly which may be due to the shape anisotropy of the particles as is evident from the HRTEM.

In order to check the stability and ageing issues of these nanoparticles, the XRD patterns were recorded after more than one year of their preparation (see ESI Fig. S1†). Here we would like to emphasize that the XRD patterns for the IO nanoparticles remains intact even after a period of one year. This is quite interesting because not only $\alpha$-Fe phase, but also iron oxide nanoparticles are quite prone to the air but showing no ageing effect in the present study. Since IO nanoparticles were surface functionalized by organic molecules $(\sim 1.0 \mathrm{~nm})$ of oleic acid, the presence of the organic canopy prevented both the direct particle contacts as well as their further oxidation. 
From the structural analysis, we observed that nanoparticles consist multiphase of iron oxide and hence probably their overall magnetic contribution is very complex. To understand their static magnetic behavior, the magnetization versus temperature measurements have been carried out in standard ZFC and FC protocol (see Fig. 4). Here we would like to mention that the low field $M_{\mathrm{ZFC}-\mathrm{FC}}$ curves for all these samples exhibit room temperature ferromagnetic ordering with a peak in ZFC/ FC magnetization for IO125, IO150, and IO200 samples at $\sim 125 \mathrm{~K}$, which is a hallmark of Verwey transition for the nonstoichiometric $\mathrm{Fe}_{3} \mathrm{O}_{4}$. However, no such peak was found for IO250. Additionally, we have also observed the presence of Morin transition ${ }^{\mathbf{1 7}}$ close to $300 \mathrm{~K}$ for IO125, IO150, IO200 samples, but not for the IO250 sample. This probably confirms the traces $\alpha-\mathrm{Fe}_{2} \mathrm{O}_{3}$ for IO125, IO150, IO200 samples, but completely ruled out for IO250 sample. It has also been observed that the nature and shape of the $M_{\mathrm{ZFC}-\mathrm{FC}}$ curves for IO125 and IO150 are very similar to each other with irreversibility temperature of $\sim 220 \mathrm{~K}$ and $300 \mathrm{~K}$ respectively. This can be attributed to a slight increase in the anisotropy contribution due to the presence of non-uniform particle geometries as well as presence of more amount of $\alpha$-Fe in case of IO150. However, in case of IO200, the shape of the curve changes drastically with irreversibility temperature much higher than the room temperature. It can be attributed to the presence of well-defined and different shaped particles (spherical, triangular, capsule, rectangle and hexagonal) that probably give a wide anisotropy distribution for this sample.

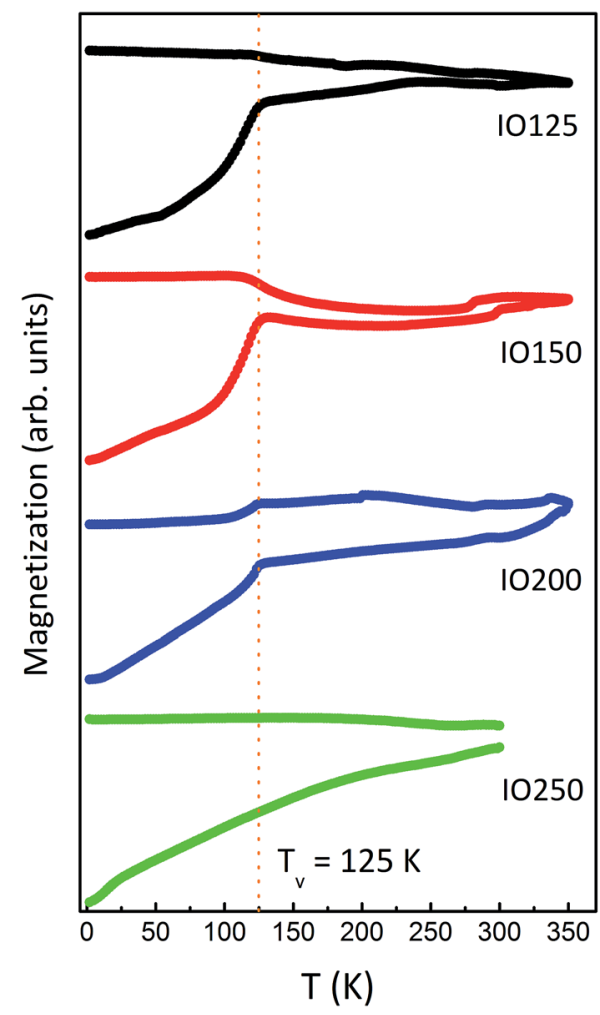

Fig. 4 Magnetization versus temperature in $\mathrm{ZFC}$ and $\mathrm{FC}$ modes at $\mathrm{H}=$ 50 Oe for 10 nanoparticles oxidized at $125^{\circ} \mathrm{C}, 150{ }^{\circ} \mathrm{C}, 200{ }^{\circ} \mathrm{C}$ and $250{ }^{\circ} \mathrm{C}$ for 30 minutes during cooling from $315^{\circ} \mathrm{C}$.
The observed behavior well corroborated with the field dependent magnetization hysteresis curve taken at $300 \mathrm{~K}$, where the sample exhibits a well-defined coercivity, whereas IO125 and IO150 are showing superparamagnetic behavior (see Fig. 5). Here we would like to mention that the saturation magnetiza-

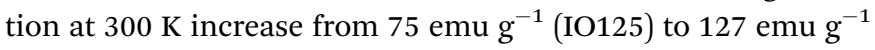
(IO150), which can be attributed to the more amount of $\alpha$-Fe as compared to IO125. The variation in magnetization can also be attributed to finite-size effect. On the other hand, IO200 and IO250 samples possess much lower values of saturation magnetization, which can also be due to the absence of $\alpha$-Fe in these two samples. Also, due to the more soft curvature for IO250 sample we could expect some other magnetization mechanism, like vortex or wall movement, and not only coherent spin rotation with field. This can also be observed in the field dependence magnetization hysteresis taken at $2 \mathrm{~K}$.

\subsection{Au-iron oxide nanoparticles: structural and magnetic characterizations}

Fig. 6-8 shows the representative TEM images for the AIO nanoparticles oxidized at $125^{\circ} \mathrm{C}, 150{ }^{\circ} \mathrm{C}, 200{ }^{\circ} \mathrm{C}$ and $250{ }^{\circ} \mathrm{C}$ for 30 minutes during cooling from the final refluxing temperature at $315{ }^{\circ} \mathrm{C}$. The only difference in the synthesis of AIO
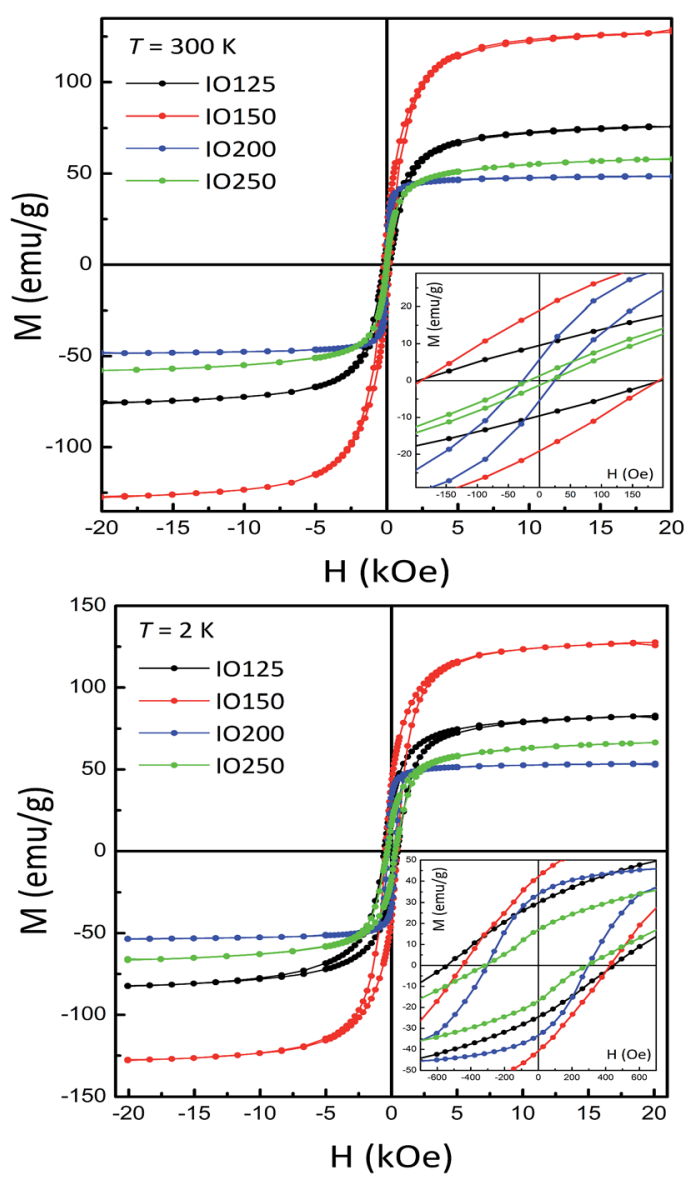

Fig. 5 Hysteresis loops recorded at (upper panel) $300 \mathrm{~K}$ and (lower panel) $2 \mathrm{~K}$ of the powder iron-oxide nanoparticles in the standard ZFC mode. 


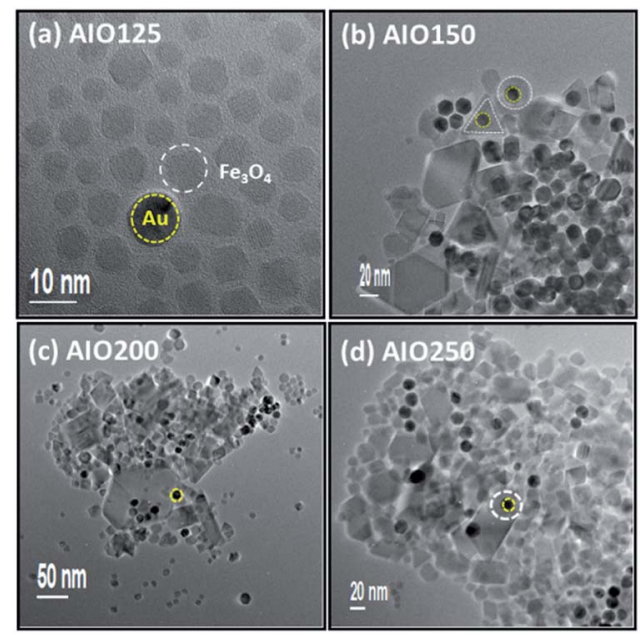

Fig. 6 TEM images of Au-iron oxide nanoparticles (AIONPs) oxidized at (a) $125^{\circ} \mathrm{C}$, (b) $150{ }^{\circ} \mathrm{C}$, (c) $200^{\circ} \mathrm{C}$ and (d) $250^{\circ} \mathrm{C}$ for 30 minutes during cooling from $315^{\circ} \mathrm{C}$.

nanoparticles is that the iron precursor is loaded in the three neck flask along with a known amount of Au nano seeds of size $\sim 14 \pm 1.0 \mathrm{~nm}$.

For AIO125, a noticeable difference in the size as well as in the shape of the particle is observed (Fig. 6a). It seems that $\mathrm{Au}$ nano seeds help and acts as a self-organization mediator and growth stabilizer agent probably through electrostatic interaction during the synthesis, hence improving the sizes distribution as well shape control of the iron oxide nanoparticle part. It is also interesting to note that the $\mathrm{Au}$ and $\mathrm{IO}$ nanoparticles are mostly phase segregated when oxidized at $125{ }^{\circ} \mathrm{C}$. The corresponding HRTEM images show the various shaped (flower type, triangular, spherical, cube, rectangle, hexagonal, capsule etc.) crystalline nanoparticles (see $\mathrm{ESI}_{\dagger} \dagger$ ).

It is observed that with the increase in the oxidation temperature halt from $125{ }^{\circ} \mathrm{C}$ to $150{ }^{\circ} \mathrm{C}$, the morphology of the particles changed and consist of some spherical-faceted and spherical triangular core-shell Au-iron oxide nanoparticles (see Fig. 6b). For AIO200, clearly heterodimer nanostructures with spherical as well as triangular shape along with a clear distinction between $\mathrm{Au}$ and iron oxide phase were observed (see Fig. 7c). Finally, for AIO250, the morphology of these nanoparticles is changed to flower type structure (see Fig. 7d).

The crystallographic structure of AIO150 spherical-triangular core-shell nanoparticles was investigated by recording its fast Fourier transform (FFT) pattern. It was clearly observed in situ nucleation and epitaxial growth of $\mathrm{Au}$ nano seeds on the spinel iron oxide $\left(\mathrm{Fe}_{3} \mathrm{O}_{4}\right.$ or $\left.\gamma-\mathrm{Fe}_{2} \mathrm{O}_{3}\right)$ nanocrystals. HRTEM of the sample AIO150 show that Au and iron oxide are in cube-on-cube epitaxy. This epitaxial growth is thermodynamically more favorable due to the lattice parameters of the inverse spinel $\mathrm{Fe}_{3} \mathrm{O}_{4}$ or $\gamma-\mathrm{Fe}_{2} \mathrm{O}_{3}(0.840-0.835 \mathrm{~nm})$ and single face centered cubic gold structure $(0.408 \mathrm{~nm})$. Fig. $8 \mathrm{~b}$ shows the FFT calcu-

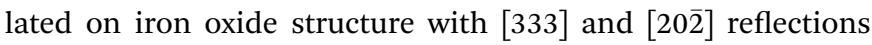
along [101] zone axis of inverse spinel $\mathrm{Fe}_{3} \mathrm{O}_{4}$ or $\gamma-\mathrm{Fe}_{2} \mathrm{O}_{3}$, whereas the Fig. $8 \mathrm{c}$ is the FFT of superimposed iron oxide and gold structures, here, [220] and [111] reflections of $\mathrm{Au}$ is

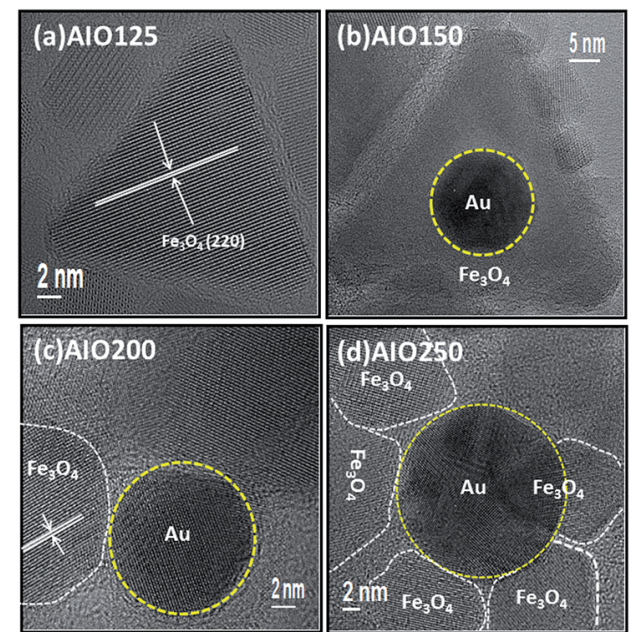

Fig. 7 HRTEM images of Au-iron oxide nanoparticles (AIONPS) oxidized at (a) $125^{\circ} \mathrm{C}$, (b) $150{ }^{\circ} \mathrm{C}$, (c) $200{ }^{\circ} \mathrm{C}$, and (d) $250{ }^{\circ} \mathrm{C}$ for 30 minutes during cooling from $315^{\circ} \mathrm{C}$.
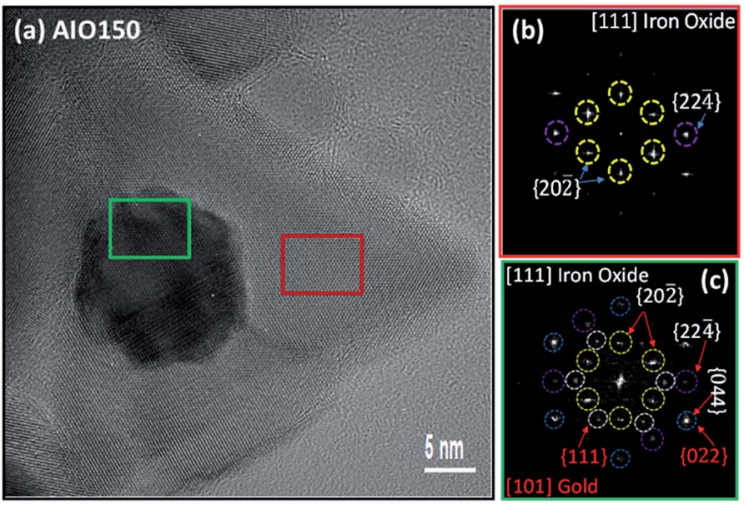

Fig. 8 HRTEM image and their corresponding FFTs calculated at different positions for AIO150 nanoparticles along with corresponding FFT patterns of the spherical-triangular core-shell.

superimposed with [440] and [222] of iron oxide. The overlapping of reflections clearly indicates the cube-on-cube epitaxial relationship between the two structures.

Fig. 9 shows the XRD pattern for the Au-iron oxide nanoparticles oxidized at $125{ }^{\circ} \mathrm{C}, 150{ }^{\circ} \mathrm{C}, 200{ }^{\circ} \mathrm{C}$ and $250{ }^{\circ} \mathrm{C}$ for 30 minutes during cooling from the final refluxing temperature at $315{ }^{\circ} \mathrm{C}$. For comparison, the XRD pattern for the Au nanoparticle seeds is also given. For all the samples, the diffraction peaks are mainly from both $\left(\mathrm{Fe}_{3} \mathrm{O}_{4} \mathrm{JCPDF} \#\right.$ 85-1436 and/or $\gamma$ $\mathrm{Fe}_{2} \mathrm{O}_{3}$ JCPDF \# 39-1346) and $\mathrm{Au}$ (JCPDF \# 04-0784). The observed peak positions remain intact, but the broadening of peaks decreased from AIO125 to AIO250, which clearly show the role of oxidizing temperature on the size (or probably on the shape as well) of $\mathrm{Au}$-iron oxide nanoparticles. However, it is interesting to observe that shape of these nanoparticles (see the HRTEM imaging) is quite different when Au nanoparticles are used as seeds to grow $\mathrm{Au}$-iron oxide nanoparticles, in comparison to the iron oxide nanoparticles. 


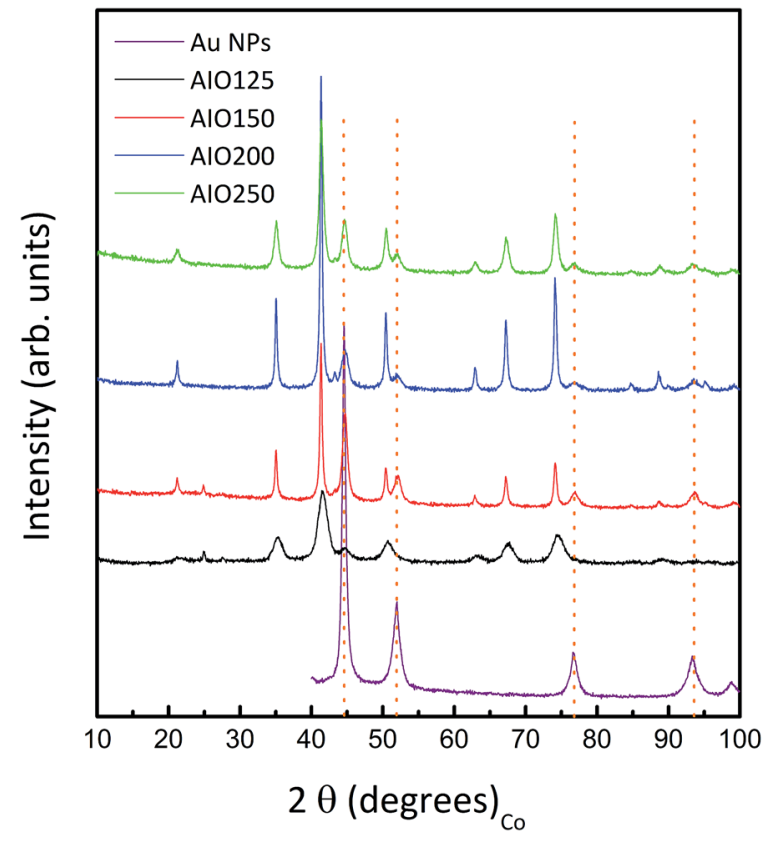

Fig. 9 X-ray diffraction (XRD) patterns of the Au-iron oxide nanoparticles oxidized at $125^{\circ} \mathrm{C}, 150^{\circ} \mathrm{C}, 200^{\circ} \mathrm{C}$ and $250{ }^{\circ} \mathrm{C}$ for 30 minutes during cooling from $315^{\circ} \mathrm{C}$.

As mentioned above, the presence of Au nano seeds help and acts as a self-organization mediator and growth stabilizer agent through electrostatic interaction during the synthesis, improving the sizes distribution and shape control of the iron oxide part. This fact can be clearly seen through the $M_{\mathrm{ZFC}-\mathrm{FC}}$ versus $T$ measurements for the samples AIO125, AIO150, AIO200 and AIO250 in an external field of 50 Oe (see Fig. 10).

Here the low field $M_{\mathrm{ZFC}-\mathrm{FC}}$ curves for the AIO125 sample exhibit a blocking process typical of an assembly of weaklyinteracting randomly oriented nanoparticles, where the $M_{\mathrm{ZFC}}$ show a narrow maximum at $\sim 57 \mathrm{~K}$, which is associated with the mean blocking temperature $\left\langle T_{\mathrm{B}}\right\rangle$ of an assembly of SPM particles. On the other side, the AIO150 display a broad maximum in the $M_{\mathrm{ZFC}}$ at $60 \mathrm{~K}$, which is related to the onset of superparamagnetic temperature. However, any fingerprint of the Verwey transition overlaps the blocked-un-blocked transition evidencing the existence of biggest iron oxide (magnetite) nanoparticles. For AIO200, the T-dependent magnetization is governed by the Verwey transition related to more amounts of the bigger magnetite nanoparticles and possible due to less contribution of smaller metallic component. In contrary, for the AIO250, magnetic behavior is very different from the other samples, which is probably due to presence of maghemite. However, all the samples have irreversibility temperature above room temperature, showing that the samples are near, or on superparamagnetic like regime close to room temperature.

From the observed hysteresis curve at $300 \mathrm{~K}$, we have noticed zero coercivity for AIO125, whereas the other samples exhibited a non-zero coercivity evidencing the presence of some amount of nanoparticles at blocking regimes. On the other hand, the hysteresis loops at $2 \mathrm{~K}$ evidenced a clear ferrimagnetic behavior for all the AIO nanoparticles (see Fig. 11). Further, the gradual

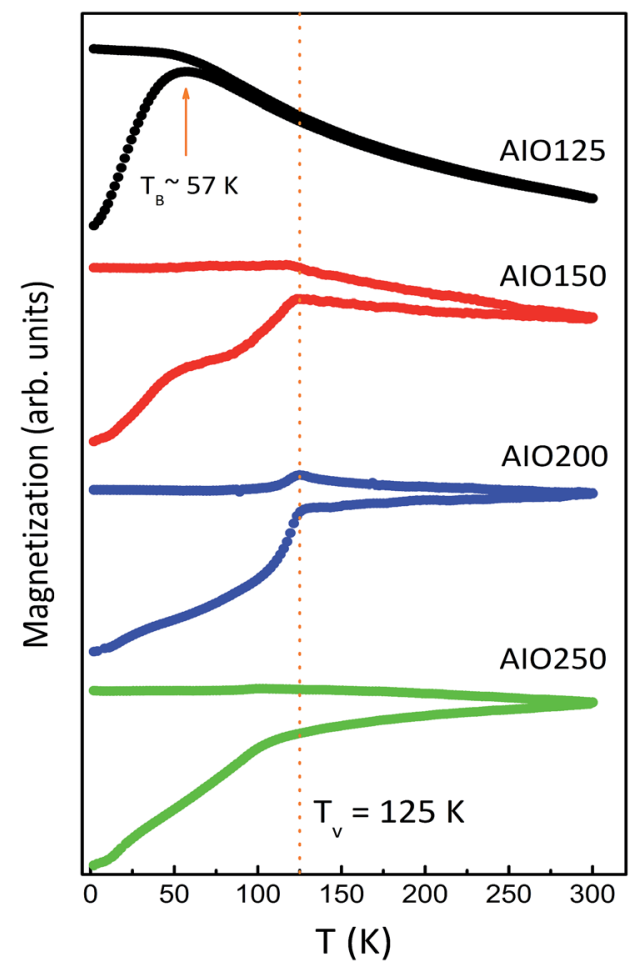

Fig. 10 Magnetization versus temperature in the standard ZFC and FC modes at $\mathrm{H}=50$ Oe for the Au-iron oxide nanoparticles.

increase of blocking temperature with cooling temperature, neglecting any anisotropy increase due others metallic phases, and is related to sizes effect.

\subsection{Mössbauer spectroscopy}

Generally, it is very difficult to distinguish between magnetite and maghemite due their similar crystal structure; therefore we have performed Mössbauer spectroscopy to distinguish the different possible phases of IO nanoparticles. Indeed, the ${ }^{57} \mathrm{Fe}$ Mössbauer spectra are sensitive to the local environment of the iron atoms. The hyperfine parameters (the isomer shift $\delta$, the quadrupole shift $\varepsilon$, and the magnetic splitting $B_{\mathrm{hf}}$ ) provide information about the electronic density and its symmetry, and also about the magnetic field experienced by the probe nucleus. The Mössbauer parameters allow the identification of nonequivalent iron positions in a crystal lattice and also the identification of compounds and phases. ${ }^{18-21}$ The room temperature Mössbauer spectra of the IO and AIO nanoparticle samples are shown in Fig. 12 and 13, respectively.

The spectra for both IO and AIO series present $\mathrm{Fe}_{3} \mathrm{O}_{4}$ as the dominating phase. However, a few presents minor phases of $\alpha$ Fe and paramagnetic $\mathrm{Fe}(\mathrm{II})$ and $\mathrm{Fe}(\mathrm{III})$. $\mathrm{Fe}_{3} \mathrm{O}_{4}$ phase was fitted with two well resolved magnetic subspectra with magnetic hyperfine fields $\left(B_{\mathrm{hf}}\right)$ of $49.2 \pm 0.1$ and $46.1 \pm 0.1 \mathrm{~T}, \delta$ of $0.281 \pm$ 0.005 and $0.663 \pm 0.005 \mathrm{~mm} \mathrm{~s}^{-1}$, and nearly null quadruple shift $(\varepsilon)$, in good agreement with bulk $\mathrm{Fe}_{3} \mathrm{O}_{4}$ parameters. ${ }^{18}$ The narrower sextet with the larger $B_{\mathrm{hf}}$ and the smaller $\delta$ is associated with high spin $\mathrm{Fe}^{3+}$ ions occupying the A-sites, while the other one is assigned to $\mathrm{Fe}^{3+} / \mathrm{Fe}^{2+}$ ions occupying the B-sites. 

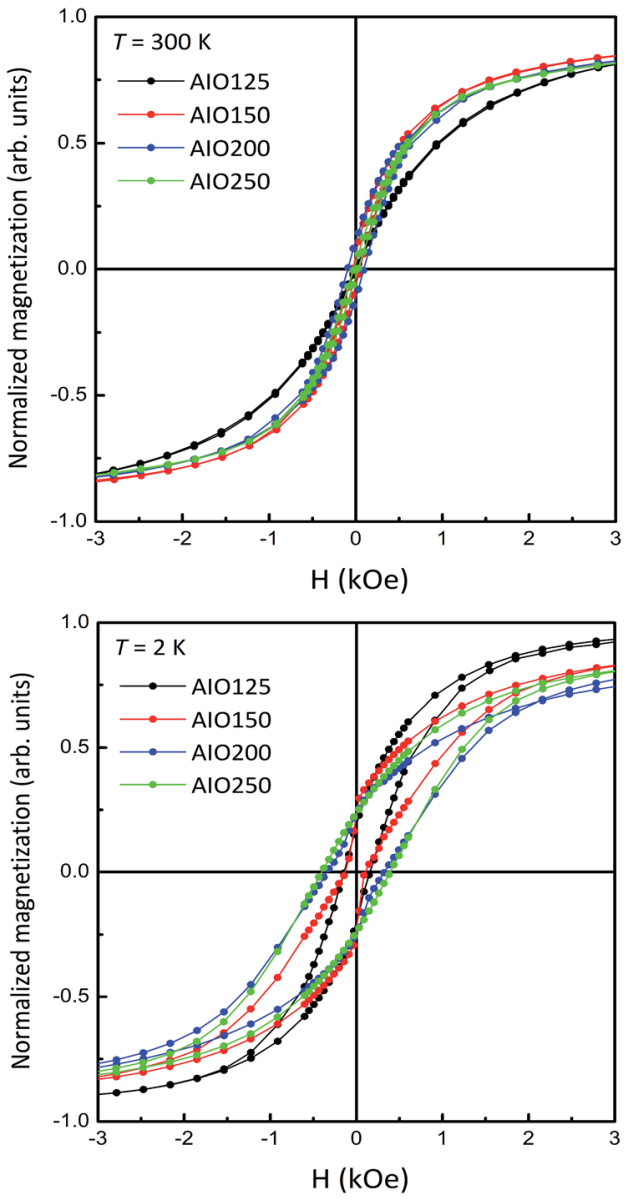

Fig. 11 Hysteresis loops recorded at (upper panel) $300 \mathrm{~K}$ and (lower panel) $2 \mathrm{~K}$ for Au-iron oxide nanoparticles.
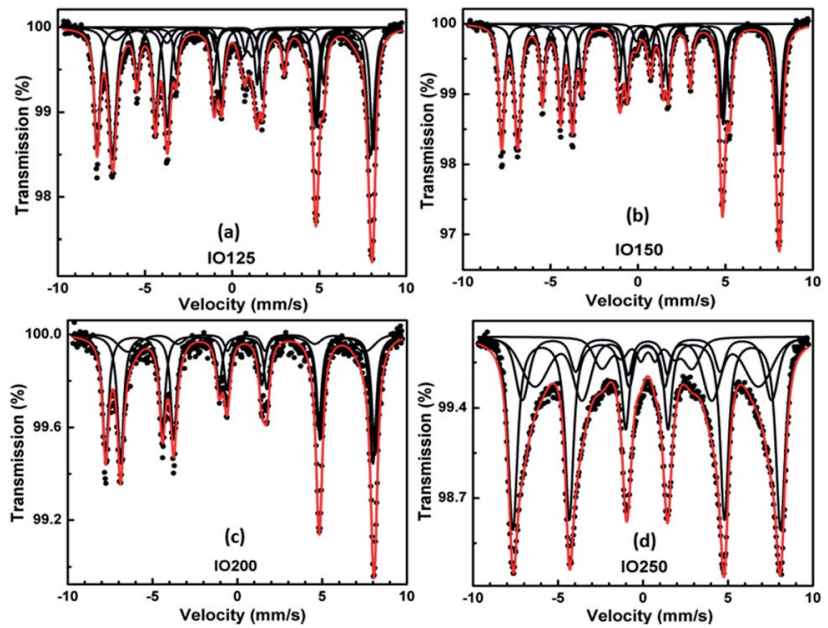

Fig. 12 Mössbauer spectrum of IONPs performed at $300 \mathrm{~K}$.

These subspectra were fitted by assuming Voigtian line shapes (Lorentzian lines of width, $\Gamma$ and Gaussian distributions of variable dispersion, $\sigma$ ).

In some samples we observed that the $B_{\mathrm{hf}}$ distribution of both sites of $\mathrm{Fe}_{3} \mathrm{O}_{4}$ broadens as a consequence of the collective
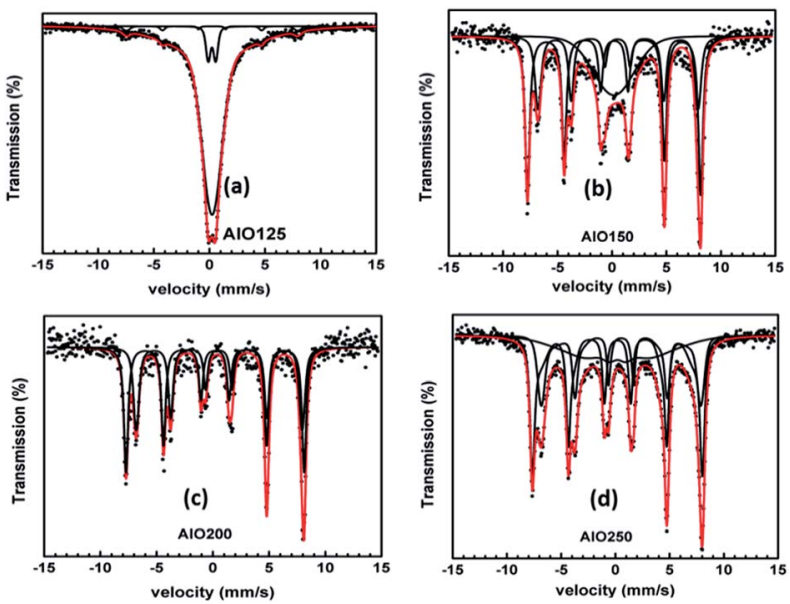

Fig. 13 Mössbauer spectrum of AIONPs performed at $300 \mathrm{~K}$.

magnetic excitations. In the absence of an applied magnetic field, the magnetization direction of a large single-domain particle is along an easy direction. However, since the anisotropy energy decreases as the particle size decreases, the thermal energy may become comparable to the anisotropy energy of a small particle. In such a case, its magnetic moment may fluctuate during the measurement process. Fluctuations can be divided into collective magnetic excitations (small amplitude fluctuation around an easy direction) and superparamagnetic relaxations (magnetization direction fluctuation among the easy directions). ${ }^{19,21}$ When the superparamagnetic relaxation time (the average time the magnetic moment remains along one easy direction between consecutive jumps) is long compared to the observation time, the magnetization vector remains close to one of the easy directions during the observation time. If the correlation time of the collective magnetic excitations is short compared to the Mössbauer observation time (the nuclear Larmor precession time), the magnetic splitting of the Mössbauer spectrum is proportional to the average value of the hyperfine field. Thus, the magnetic splitting of a nanoparticle Mössbauer spectrum is generally smaller than that of a macroscopic crystal, and depends on the particle volume. If a sample contains nanoparticles with size distribution, the magnetic splitting in particles of different volumes will be different. This results in a broadening of the Mössbauer lines.

To take into account the collective magnetic excitations, the spectra that presents this phenomena were fitted by assuming additional subspectra restricted to have equal hyperfine parameters to the main A-sites and B-sites contributions of $\mathrm{Fe}_{3} \mathrm{O}_{4}$ unless $B_{\mathrm{hr}}$. The observed magnetic hyperfine fields values are lower than the bulk one, reflecting the collective magnetic excitations. AIO125 is the sample with lowest size $(\sim 9 \mathrm{~nm})$ in AIO series and consistently is the unique sample that presents superparamagnetic relaxation in the Mössbauer spectrum ( $\sim 2 \%$ of the area fraction). This sample also presents $50 \%$ of the area fraction with collective magnetic excitations phenomena. IO250 sample presents the higher area fraction $(\sim 53 \%)$ with collective magnetic excitations phenomena in the 
IO series in accordance with the TEM results ( $\sim 10 \mathrm{~nm})$. IO200 sample presents only $13 \%$ of the area fraction with collective magnetic excitations phenomena in agreement with its nanoparticle size $(\sim 12 \mathrm{~nm})$, whereas AIO250 sample presents $47 \%$ of the area fraction with collective magnetic excitations phenomena similarly to IO250 sample in accordance to their particle sizes. On the other hand, IO125, IO150 and AIO200 samples have big enough particle sizes and therefore no collective Fig. 13 Mössbauer spectrum of $\mathrm{Au}$-iron oxide nanoparticles performed at $300 \mathrm{~K}$.

The whole sets of IO and AIO series presents area fractions of superparamagnetic behavior plus collective magnetic excitations consistently with the nanoparticle size of each sample, as is shown in Fig. 14. This area fraction is plotted as function of the nanoparticle size in Fig. 14.

As mentioned before, some of the samples present minor phase: $\alpha$-Fe $(\sim 14 \%$ in IO 125 and $\sim 21 \%$ in IO150), Fe(II) $(\sim 4 \%$ in IO 125$)$ and $\mathrm{Fe}$ (III) ( $\sim 2 \%$ in IO 150 and $\sim 6 \%$ in AIO 125). However, increase in the oxidation temperatures resulted with only magnetite $\left(\mathrm{Fe}_{3} \mathrm{O}_{4}\right)$ phase without any traces of paramagnetic Fe(II) and Fe(III) as observed for IO200, IO250, AIO150, $\mathrm{AIO} 200$ and $\mathrm{AIO} 250$.

IO125 and IO150 display well resolved magnetic subspectra with magnetic hyperfine fields of $33.2 \pm 0.1 \mathrm{~T}$, isomer shift of $0.005 \pm 0.005 \mathrm{~mm} \mathrm{~s}^{-1}$ and null quadruple shift, which is in a good agreement with bulk $\alpha$-Fe parameters. ${ }^{13}$ The area fractions of these subspectra are $\sim 13.4 \%$ and $\sim 21 \%$ for IO125 and IO150 samples, respectively, whereas for the other samples, no subspectra appeared from $\alpha$-Fe. IO125 presents non-magnetic sub-spectrum with isomer shift of $0.98 \pm 0.02 \mathrm{~mm} \mathrm{~s}^{-1}$ and quadruple shift of $0.59 \pm 0.02 \mathrm{~mm} \mathrm{~s}^{-1}$, corresponding to paramagnetic $\mathrm{Fe}^{2+}$. The area fraction of this sub-spectrum is $\sim 4 \%$, whereas IO150 and AIO125 also present a non-magnetic subspectra with area fractions of $\sim 2 \%$ and $\sim 6 \%$ respectively, corresponding to paramagnetic $\mathrm{Fe}^{3+}$ with isomer shifts of 0.38 $\pm 0.02 \mathrm{~mm} \mathrm{~s}^{-1}$ and $0.35 \pm 0.02 \mathrm{~mm} \mathrm{~s}^{-1}$, and quadruple shifts of $0.73 \pm 0.02$ and $0.67 \pm 0.02$, respectively.

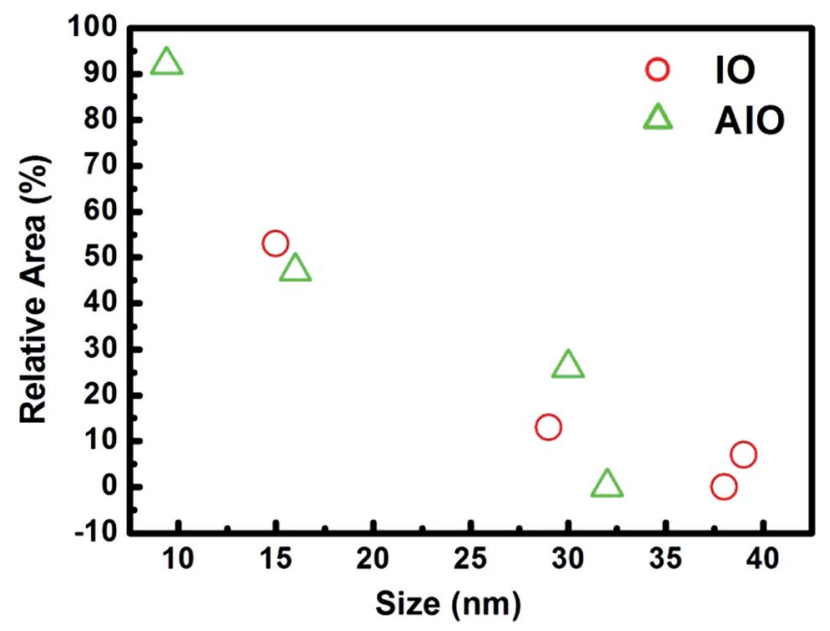

Fig. 14 Mössbauer area fractions of subspectra corresponding to superparamagnetic plus collective magnetic excitations behavior.

\subsection{Magneto-hyperthermia study}

Magnetic hyperthermia is a therapeutic procedure which relies on the ability of magnetic nanoparticles to absorb energy from an alternating magnetic field and release it as heat. This is a low invasive therapy, where a notable lack of side effects makes it an attractive therapy, alone or by improving the efficiency of chemotherapy and radiation. ${ }^{22,23}$ Food and Drug Administration (FDA) belonging to the USA has approved the iron oxide nanoparticles for a limited use in theranostic applications, such as in hyperthermia application.

Quantification of the power dissipation of magnetic nanoparticles is usually done by measuring specific absorption rate (SAR). A high heating potential is crucial for the therapeutic use, because it would require a smaller amount of nanoparticles to be injected to the patient. The SAR of the nanoparticles in the fluid was retrieved from measurements of temperature $(T)$ of the ferrofluid as function of time $(t)$, while an alternating magnetic field was applied. SAR of the nanoparticles in the fluid was calculated from the measured slopes, $\Delta T / \Delta t$ using the expression: ${ }^{24}$

$$
\operatorname{SAR}\left(\mathrm{W} \mathrm{g}^{-1}\right)=\frac{C}{\phi} \Delta T / \Delta t, \quad \phi=\frac{m_{\mathrm{NP}}}{m_{\mathrm{FF}}}
$$

where $C$ is the FF specific heat (here taken as that of hexane: $\left.2.015 \mathrm{~J} \mathrm{~g}^{-1} \mathrm{~K}^{-1}\right), m_{\mathrm{FF}}$ is the mass of the ferrofluid and $m_{\mathrm{NP}}$ is the mass of the magnetic nanoparticles in the Dewar. We have selected two samples namely IO200 and AIO125 for the magnetic fluid hyperthermia study with different shape and morphology. To obtain stable ferrofluids IO200 and AIO125 nanoparticles were suspended in hexane and the ferrofluids were labeled as FFIO200 and FFAIO125 with concentrations 9.8 $\mathrm{mg} \mathrm{ml}^{-1}$ and $4.6 \mathrm{mg} \mathrm{ml}^{-1}$, respectively.

The results of three independent time-dependent calorimetric measurements for FFIO125 ferrofluid are shown in Fig. 15. For this sample the slope $\Delta T / \Delta t$ obtained is $0.224 \pm$ $0.008 \mathrm{~K} \mathrm{~s}^{-1}$. SAR value obtained for IO200 is $64 \pm 2 \mathrm{~W} \mathrm{~g}^{-1}$ and for AIO125 is $5 \pm 2 \mathrm{~W} \mathrm{~g}^{-1}$. It is important to highlight that the

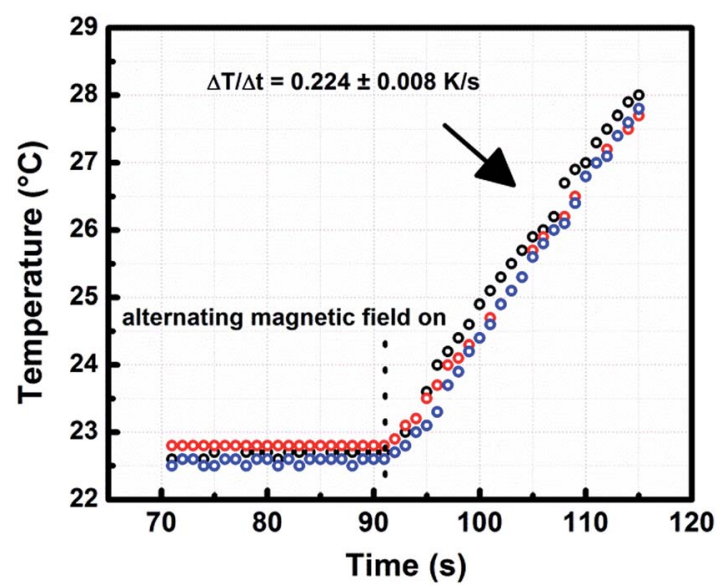

Fig. 15 Temperature increase due to the heating of $0.5 \mathrm{ml} \mathrm{FFIO200}$ ferrofluid in an alternating magnetic field with frequency of $255 \mathrm{kHz}$ and amplitude of $35 \mathrm{kA} \mathrm{m}^{-1}$. Three independent measurements are shown. 
SAR value is expressed per mass of nanoparticles, and in the case of AIO a mass relation $3: 1$ is estimated for Au: magnetite. So, the SAR value of AIO125 expressed per mass of magnetic nanoparticle will be approximately four times higher than the value reported. There are two relaxation mechanisms by which single-domain magnetic nanoparticles may generate thermal energy: Néel relaxation and Brownian relaxation. These processes correspond to the nanoparticle magnetic moment switching among its easy axis directions and the viscous friction due to the Brownian rotational diffusion of particles in the fluid, respectively. Brownian relaxation is dominant in larger particles and Néel relaxation is dominant in smaller particles; the size region where both mechanisms occur simultaneously depends strongly on the anisotropy constant of the particle and temperature $\mathrm{e}^{25}$ as well as on the viscosity of the ferrofluid. At the experimental condition of our measurements and considering typical anisotropy values of magnetite nanoparticles, ${ }^{26,27}$ the crossover from Neel to Brown relaxation occurs at nanoparticle diameters between 12 and $20 \mathrm{~nm}$. Therefore AIO125 sample relax through Néel mechanism meanwhile IO200 sample relax by Brown mechanism. In spite of the difference in the mechanisms of relaxation in both sample, the SAR values are determine principally by the nanoparticle size. SAR is monotonically increasing function of nanoparticle size. ${ }^{28,29}$ This is the reason for why the IO200 samples presents a higher SAR value than AIO125, despite it presents lower saturation magnetization.

\section{Conclusions}

Iron oxide and $\mathrm{Au}$-iron oxide nanocomposites synthesized by thermal decomposition method offers not only the Au-iron oxide core-shell system with controlled size and varying shape but also long term stabilization. Crystallographic and magnetic studies show the strong dependence of magnetic properties on particle morphology (or shape anisotropy). Increase in temperature as well as oxidation time results in fully grown core-shell nanoparticles with magnetite phase with spinel structure as major phase. The presence of magnetite was further confirmed by the Verwey transition and Mössbauer spectroscopy technique. The calorimetric study highlights the potential of these nanoparticles for hyperthermia application and the importance of shape anisotropy on the SAR values.

\section{Acknowledgements}

SKS is grateful to FAPEMA for providing financial support. Sarveena would like to thank UGC-DAE CSR, Indore Centre for financial support under CRS Scheme. J. M. would like to acknowledge the full support by Conicet. O. M. L. acknowledges FAPESP grant 2014/26672-8.

\section{References}

1 H. Yu, M. Chen, P. M. Rice, S. X. Wang, R. L. White and S. Sun, Nano Lett., 2005, 5, 2; S. K. Sharma, J. M. Vargas, S. Kumar, C. G. Lee, K. R. Pirota and M. Knobel, J. Alloys Compd., 2011, 509, 6414.
2 K. C. F. Leung, S. Xuan, X. Zhu, D. Wang, C. P. Chak, S. F. Lee, W. K. W. Ho and B. C. T. Chung, Chem. Soc. Rev., 2012, 41, 1911.

3 P. K. Jain, X. Huan and M. A. El-Sayed, Plasmonics, 2007, 2, $107 \mathrm{e} 18$.

4 Wahajuddin and S. Arora, Int. J. Nanomed., 2012, 7, 3445.

5 Z. Bao, Z. Sun, Z. Li, L. Tian, T. Ngai and J. Wang, Langmuir, 2011, 27, 5071.

6 E. V. Shevchenko, M. I. Bodnarchuk, M. V. Kovalenko, D. V. Talapin, R. K. Smith, S. Aloni, W. Heiss and A. Paul Alivisatos, Adv. Mater., 2008, 20, 4323.

7 E. N. Esenturk and A. R. Hight Walker, J. Nanopart. Res., 2013, 15, 1364.

8 F. Pineider, C. D. J. Fernandez, V. Videtta, E. Carlino, A. Al Hourani, F. Wilhelm, A. Rogalev, P. D. Cozzoli, P. Ghigna and C. Sangregorio, ACS Nano, 2013, 7, 857.

9 S. V. Salihov, Y. A. Ivanenkov, S. P. Krechetov, M. S. Veselov, N. V. Sviridenkova, A. G. Savchenko, N. L. Klyachko, Y. I. Golovin, N. V. Chufarova, E. K. Beloglazkina and A. G. Majouga, J. Magn. Magn. Mater., 2015, 394, 173.

10 L. Wang, J. Luo, Q. Fan, M. Suzuki, I. S. Suzuki, M. H. Engelhard, Y. Lin, N. Kim, J. Q. Wang and C. Jian Zhong, J. Phys. Chem. B, 2005, 109, 21593; S. K. Sharma, J. M. VargasN, M. Vargas, S. C. Sepúlveda, D. Altbir, K. R. Pirota, R. Zboril, G. Zoppellaro and M. Knobel, RSC $A d v .$, 2015, 5, 17117.

11 M. B. Gawande, A. Goswami, T. Asefa, H. Guo, A. V. Biradar, D. L. Peng, R. Zboril and R. S. Varma, Chem. Soc. Rev., 2015, 44, 7540 .

12 R. G. Chaudhuri and S. Paria, Chem. Rev., 2012, 112, 2373.

13 A. Gole, J. W. Stone, W. R. Gemmill, H. C. ZurLoye and C. J. Murphy, Langmuir, 2008, 24, 6232.

14 H. Yu, M. Chen, P. M. Rice, S. X. Wang, R. L. White and S. Sun, Nano Lett., 2005, 5, 379.

15 J. Choi, S. Park, Z. Stojanović, H. S. Han, J. Lee, H. K. Seok, D. Uskoković and K. Hyi Lee, Langmuir, 2013, 29, 15698.

16 B. D. Cullity, Elements of X-ray Diffraction, Addison-Wesely, Reading, MA, 1987.

17 J. Wang, V. Aguilar, L. Li, F. Li, W. Z. Wang and G. M. Zhao, Nano Res., 2015, 8, 1906.

18 R. E. Vandenberghe, C. A. Barrero, G. M. D. Costa, E. V. San and E. D. Grave, Hyperfine Interact., 2000, 126, 247.

19 S. Morup and H. Tops, Appl. Phys., 1976, 11, 63.

20 R. Zboril, M. Mashlan and D. Petridis, Chem. Mater., 2002, 14, 969.

21 R. L. Cohen, Applications of Mössbauer Spectroscopy II Bell Laboratories, Academic Press, New York, New Jersey, 1980.

22 A. Ito, M. Fujioka, T. Yoshida, K. Wakamatsu, S. Ito, T. Yamashita, K. Jimbow and H. Honda, Cancer Sci., 2007, 98, 424.

23 F. Gazeau, M. Levy and C. Wilhelm, Nanomedicine, 2008, 3, 831.

24 M. E. F. Brollo, J. M. O. Henao, R. L. Ruiz, D. Muraca, C. S. B. Dias, K. R. Pirota and M. Knobel, J. Magn. Magn. Mater., 2016, 397, 20.

25 A. E. Deatsch and B. A. Evans, J. Magn. Magn. Mater., 2014, 354, 163. 
26 V. Dolgovskiy, V. Lebedev, S. Colombo, A. Weis, B. Michen, L. A. Hirschi and A. P. Fink, J. Magn. Magn. Mater., 2015, 379, 137.

27 D. F. Coral, P. M. Zélis, M. E. de Sousa, D. Muraca, V. Lassalle, P. Nicolás, M. L. Ferreira and M. B. Fernández van Raap, J. Appl. Phys., 2014, 15, 043907.
28 R. E. Rosensweig, J. Magn. Magn. Mater., 2002, 252, 370.

29 M. Arana, P. G. Bercoff, S. E. Jacobo, P. M. Zélis and G. A. Pasquevich, Ceram. Int., 2016, 42, 1545. 